\title{
HeilberufeSCIENCE und HeilberufeSCIENCE-Symposium
}

\author{
Jörg Klewer
}

Online publiziert: 23. Mai 2014

(C) Springer-Verlag Wien 2014

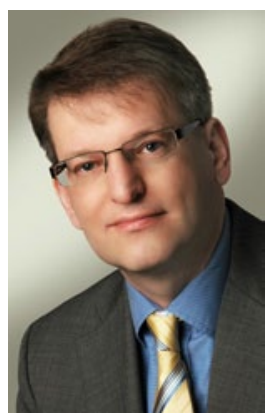

Jörg Klewer

Das HeilberufeSCIENCE-Symposium, das in den vergangenen Jahren im Rahmen des Gesundheitspflegekongresses jeweils im Herbst in Hamburg stattfand, soll zu seinen Wurzeln zurückkehren. Daher wird dieses Jahr in Hamburg kein HeilberufeSCIENCE-Symposium stattfinden, sondern erst wieder im April 2015 im Rahmen des Dresdner Pflegekongresses vom 16.-18.04.2015. Der zugehörige Call for abstracts wird in diesem Jahr daher erst im Herbst erscheinen und Sie zur Einreichung von Beitragsvorschlägen am HeilberufeSCIENCE-Symposium einladen.

In der 2. Ausgabe 2014 von HeilberufeSCIENCE freuen wir uns, Sie mit neuen wissenschaftlichen Beiträgen zu informieren. Vogt geht auf Belastungen von Mitarbeitern in der stationären Pflege durch das Miterleben von Sterben, Tod sowie Trauer ein und präsentiert die Ergebnisse einer umfangreichen schriftlichen Befragung der Mitarbeiter von 10 ausgewählten Einrichtungen der stationären Altenpflege in Deutschland. Mäding et al. haben Transplantationsbeauftragte in der Region Ost der Deutschen Stiftung Organtrans-

J. Klewer $(\bowtie)$

Zwickau, Deutschland

E-Mail: joklewer@t-online.de plantation (DSO) befragt und weisen auf der Grundlage der Ergebnisse darauf hin, dass Krankenhäuser noch intensiver auf die Belange der Transplantationsbeauftragten eingehen sollten.

Die wissenschaftlichen Kurzmitteilungen beschäftigen sich mit den Themen Depression, ,palliative care“ und muslimische Patienten. Hoffmann und Kugler zeigen exemplarisch anhand der Kasuistik eines Patienten mit unipolarer Depression, welche finanziellen Auswirkungen eine nichtleitliniengerechte Versorgung des Patienten haben kann. Bemmann et al. berichten über die Ergebnisse einer schriftlichen Befragung von 150 Altenpflegeschülern aus 4 Altenpflegeschulen zu deren Wissen und Einstellungen zu Palliative care. Dabei wurde deutlich, dass das Wissen über Palliative care bei Demenz und die Leistungsmöglichkeiten der ambulanten Hospizdienste gering war. Kronenthaler et al. haben eine schriftliche Befragung von Auszubildenden im Pflegebereich zu deren Kenntnisstand über den Islam und zu Erlebnissen mit muslimischen Patienten durchgeführt. Die Untersuchungsergebnisse zeigen, dass Wissensdefizite bezüglich des islamischen Glaubens vorhanden waren und dass eine $\mathrm{zu}$ diesem Thema durchgeführte Schulungsmaßnahme positiven Einfluss auf den Kenntnisstand hatte.

An dieser Stelle möchte ich Sie wieder einladen, die aktuellen Beiträge dieser Ausgabe von HeilberufeSCIENCE interessiert und kritisch zu lesen - und natürlich würde es mich freuen, wenn ich Sie in der August-Ausgabe von HeilberufeSCIENCE im Sommer wieder an dieser Stelle begrüßen darf.

Ihr

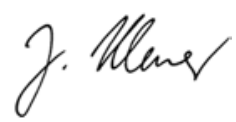

